\title{
OPEN Direct detection of SARS-CoV-2 using non-commercial RT-LAMP reagents on heat-inactivated samples
}

\author{
Alisa Alekseenko $\mathbb{( 1 0}^{1,9}$, Donal Barrett $\mathbb{C}^{1,9}$, Yerma Pareja-Sanchez $\mathbb{1}^{1,9}$, Rebecca J. Howard $\mathbb{1}^{2}$, \\ Emilia Strandback ${ }^{3}$, Henry Ampah-Korsah $\mathbb{1}^{3}$, Urška Rovšnik $\mathbb{1}^{2}$, Silvia Zuniga-Veliz ${ }^{4}$, \\ Alexander Klenov ${ }^{5}$, Jayshna Malloo ${ }^{5}$, Shenglong Ye ${ }^{6}$, Xiyang Liu ${ }^{6}$, Björn Reinius $\circledR^{3}$, \\ Simon J. Elsässer ${ }^{7}{ }^{7}$, Tomas Nyman ${ }^{3}$, Gustaf Sandh ${ }^{4}$, Xiushan Yin ${ }^{1,6,8}$ \& \\ Vicent Pelechano ${ }^{1}{ }^{1 \bowtie}$
}

RT-LAMP detection of SARS-CoV-2 has been shown to be a valuable approach to scale up COVID19 diagnostics and thus contribute to limiting the spread of the disease. Here we present the optimization of highly cost-effective in-house produced enzymes, and we benchmark their performance against commercial alternatives. We explore the compatibility between multiple DNA polymerases with high strand-displacement activity and thermostable reverse transcriptases required for RT-LAMP. We optimize reaction conditions and demonstrate their applicability using both synthetic RNA and clinical patient samples. Finally, we validate the optimized RT-LAMP assay for the detection of SARS-CoV- 2 in unextracted heat-inactivated nasopharyngeal samples from 184 patients. We anticipate that optimized and affordable reagents for RT-LAMP will facilitate the expansion of SARS-CoV-2 testing globally, especially in sites and settings where the need for large scale testing cannot be met by commercial alternatives.

The ongoing SARS-CoV-2 pandemic has had a tremendous impact on society, surpassing one million casualties worldwide and exposing the vulnerability of our globalised world to the spread of infectious disease. Extensive testing and isolation of confirmed cases has been proposed as a viable strategy to limit the spread of SARS-CoV-2 $2^{1-3}$. Consequently, many methods for SARS-CoV-2 ribonucleic acid detection have been developed (reviewed $\mathrm{in}^{4}$ ). The gold standard for RNA detection is the reverse transcription quantitative PCR (RT-qPCR), requiring specialized equipment of limited availability in many contexts (e.g., real time thermocyclers). Due to their intrinsic simplicity and high sensitivity, isothermal detection methods are increasingly appreciated as well suited for point-of-care (POC) testing ${ }^{5}$, and among them LAMP (loop-mediated isothermal amplification) is an attractive approach ${ }^{6,7}$. Early on during the COVID-19 pandemic many researchers, including ourselves, worked to adapt and optimize RT-LAMP detection methods for SARS-CoV-2 diagnostics ${ }^{8-10}$. This initial work quickly expanded with optimized oligonucleotide designs and simplified purification systems ${ }^{11-13}$. The specificity of RT-LAMP has been improved by applying CRISPR based detection ${ }^{14,15}$ or high-throughput sequencing to confirm amplicon sequences and to facilitate mass usage ${ }^{16,17}$. Simplified isothermal approaches have the potential to facilitate SARS-CoV-2 diagnosis and thus contribute to the efforts against the COVID-19 pandemic. This is especially important when considering that frequent testing, even at a lower sensitivity, can contribute to a decrease in spread of the disease $\mathrm{e}^{1-3}$.

${ }^{1}$ SciLifeLab, Department of Microbiology, Tumor and Cell Biology, Karolinska Institutet, Tomtebodavägen

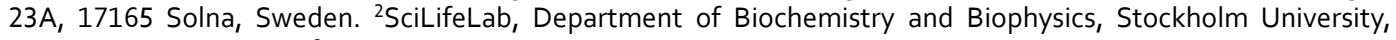
17121 Solna, Sweden. ${ }^{3}$ Department of Medical Biochemistry and Biophysics, Karolinska Institutet, Solna, Sweden. ${ }^{4}$ Department of Clinical Microbiology, Karolinska University Hospital, Stockholm, Sweden. ${ }^{5}$ Hudak Lab, Department of Biology, York University, 4700 Keele St, Toronto, ON M3J 1P3, Canada. ${ }^{6}$ Biotech and Biomedicine Science (Shenyang) Co. Ltd, Shenyang 110000, China. ${ }^{7}$ SciLifeLab, Department of Medical Biochemistry and Biophysics, Division of Genome Biology, Karolinska Institutet, Solna, Sweden. ${ }^{8}$ Applied Biology Laboratory, Shenyang University of Chemical Technology, Shenyang 110142, China. ${ }^{9}$ These authors contributed equally: Alisa Alekseenko, Donal Barrett and Yerma Pareja-Sanchez. ${ }^{\circledR}$ email: vicente.pelechano.garcia@ki.se 
However, access to reliable and affordable molecular biology reagents remains a problem. Even though the production and distribution have significantly increased since the start of the crisis, the need for testing to control disease spread and allow reopening of society keeps raising the demand. This is especially problematic in countries with limited distribution channels and a lack of economic resources to afford massive testing. To make molecular testing more accessible and allow its widespread implementation, multiple approaches have been developed. For example, it has recently been shown how a simplified RT-qPCR reaction for SARS-CoV-2 detection is feasible using only one enzyme ${ }^{18}$, or even how it is possible to use crude enzymes from lyophilized bacteria for LAMP ${ }^{19}$. Here we aim to contribute to the global effort to generate affordable SARS-CoV-2 tests based on in-house produced enzymes and thus democratize their use. We established protocols for simple production of DNA polymerases with high strand-displacement activity ${ }^{20}$ and optimized their reaction conditions for RT-LAMP. We explore their compatibility with thermostable reverse transcriptases and provide a complete in-house reagent mix for fluorescent or colorimetric detection of SARS-CoV-2 RNA. We then benchmarked the optimized reaction mix with multiple commercial alternatives and RT-LAMP primer sets. Finally, we tested the ability of the produced reagents to correctly detect SARS-CoV-2 presence directly in non-purified clinical nasopharyngeal samples from 184 patients.

\section{Materials and methods}

Expression and purification of strand displacing polymerases.. Displacement polymerases assayed for RT-LAMP polymerase activity included the Geobacillus stearothermophilus exonuclease-deficient family-A polymerase large fragment (Bst-LF), and its 'v5.9' and 'v7.16' derivatives previously reported by Ellington and colleagues to incorporate functional properties of the related Klentaq polymerase from Thermus aquaticus ${ }^{20}$. Prior to functional characterization, hexahistidine (H6)-tagged variants were screened for expression efficiency in various vectors, including pTetA (constructs kindly provided by Andrew Ellington), pET-16b and pET28a (synthesized for this work). Constructs in pET-16b contained an ASRGS-H6 followed by the polymerase sequence, inserted between NcoI and BamHI sites in the multiple cloning region. Similarly, constructs in pET28a were engineered by inserting the polymerase between NdeI and XhoI, $3^{\prime}$ to the incorporated GSS-H6-SSG and thrombin cleavage sequences.

Polymerase expression and purification protocols were adapted from that of Milligan and colleagues ${ }^{20}$. Briefly, transformed BL21(DE3) cells (Merck KGaA, Darmstadt, Germany) were cultured overnight at $37^{\circ} \mathrm{C}$, followed by inoculation (1:200) into fresh $2 x Y T$ media with suitable antibiotic $(100 \mathrm{mg} / \mathrm{L}$ ampicillin for constructs in pTetA and pET-16b, $50 \mathrm{mg} / \mathrm{L}$ kanamycin for pET-28a). After cells reached an optical density (OD600) of $0.6-0.8$, expression was induced $(200 \mu \mathrm{g} / \mathrm{L}$ tetracycline- $\mathrm{HCl}$ in $70 \%$ ethanol for pTet $\mathrm{A}, 100 \mu \mathrm{M}$ isopropyl$\beta$-D-1-thiogalactopyranoside for pET-16b or pET-28a) and cells grown for an additional $3-7 \mathrm{~h}$ at $30-37^{\circ} \mathrm{C}$ before harvesting. Pellets were resuspended, sonicated, and cleared by ultracentrifugation ( $30 \mathrm{~min}$ at $40,000 \times \mathrm{g}$, $\left.4^{\circ} \mathrm{C}\right)$ in buffer $\mathrm{A}(20 \mathrm{mM}$ Tris $\mathrm{pH} 7.4,300 \mathrm{mM} \mathrm{NaCl}, 0.1 \%$ Tween-20, $10 \mathrm{mM}$ imidazole) supplemented with $500 \mathrm{mg} / \mathrm{L}$ lysozyme and EDTA-free protease inhibitors. As an initial purification step, lysate supernatants were then heated to $65^{\circ} \mathrm{C}$ for $20 \mathrm{~min}$ with gentle shaking $(400 \mathrm{rpm})$, then again cleared by ultracentrifugation $(20 \mathrm{~min}$ at $20,000 \times g, 4^{\circ} \mathrm{C}$ ). Clarified supernatants were purified by $\mathrm{Ni}^{2+}$ affinity (HisPur Ni-NTA resin (Thermo Fisher Scientific, Waltham, MA, USA)), eluting in buffer A with $250 \mathrm{mM}$ imidazole. Positive elution fractions were pooled and concentrated in storage buffer $(10 \mathrm{mM}$ Tris pH 7.4, $100 \mathrm{mM} \mathrm{KCl,} 1 \mathrm{mM}$ DTT, $0.1 \mathrm{mM}$ EDTA, 0.5\% Tween-20, 0.5\% Triton-X100, 50\% glycerol), then aliquoted, flash-frozen in liquid nitrogen, and stored at -80 ${ }^{\circ} \mathrm{C}$. All constructs yielded at least $3 \mathrm{mg}$ purified protein per liter liquid culture, sufficient to amplify thousands of individual RT-LAMP samples at negligible consumables cost.

Expression and Purification of thermostable reverse transcriptases.. The MashUp-RT plasmid (pET backbone) (kindly provided by https://pipettejockey.com/) was transformed into BL21 (DE3) T1R pRARE2 expression strain and plated on L-Broth (LB) agar (Formedium, Norfolk, UK) plate containing $50 \mu \mathrm{g} /$ $\mathrm{mL}$ kanamycin. Overnight cultures were inoculated with fresh transformants and grown at $37^{\circ} \mathrm{C}$ at $175 \mathrm{RPM}$ in LB medium (Formedium, Norfolk, UK) containing kanamycin $(50 \mu \mathrm{g} / \mathrm{mL})$. Subsequently, the overnight culture was diluted into $1.5 \mathrm{~L}$ fresh LB medium supplemented with kanamycin and catabolite repression buffer $(25 \%$ glycerol, $25 \%$ glucose, $1 \mathrm{mM} \mathrm{MgSO}_{4}$ and $0.1 \mathrm{mM} \mathrm{MnCl}_{2}$ ) and the cultures were grown at $37^{\circ} \mathrm{C}$ in the LEX system (LEX-48, Epiphyte Three Inc., Toronto, Canada). At $\mathrm{OD}_{600} 0.9$, the cultures were down-tempered to $18^{\circ} \mathrm{C}$ before protein expression was induced with $0.5 \mathrm{mM}$ IPTG and the culture was grown additionally for $24 \mathrm{~h}$ at $18{ }^{\circ} \mathrm{C}$. The cells were harvested by centrifugation at $4500 \times g$ for $10 \mathrm{~min}$ at $4{ }^{\circ} \mathrm{C}$ and resuspended in MashUp-RT IMAC lysis buffer ( $25 \mathrm{mM}$ Tris- $\mathrm{HCl}, 300 \mathrm{mM} \mathrm{NaCl}, 10 \%$ glycerol, $40 \mathrm{mM}$ imidazole, $0.5 \%$ Triton X-100, $\mathrm{pH}$ 8) supplemented with $1 \mathrm{mg} / \mathrm{mL}$ lysozyme and one tablet Complete EDTA-free protease inhibitor cocktail (Roche Diagnostics $\mathrm{GmbH}$, Mannheim, Germany) per $1.5 \mathrm{~L}$ culture. The resuspended cell pellets were stored at $-80^{\circ} \mathrm{C}$.

The pET21 vector containing the coding sequence of RTX (reverse transcriptase) and RTX ${ }^{\text {exo-}}$ (RTX lacking exonuclease activity), kindly provided by Andy Ellington, were transformed into BL21(DE3) T1R pRARE2 expression strain and plated on LB plates containing carbenicillin $(50 \mu \mathrm{g} / \mathrm{mL})$ and chloramphenicol $(34 \mu \mathrm{g} /$ $\mathrm{mL})$. Terrific Broth (TB) medium (Merck, Darmstadt, Germany) supplemented with carbenicillin $(100 \mu \mathrm{g} / \mathrm{mL})$ and chloramphenicol $(34 \mu \mathrm{g} / \mathrm{mL}), 8 \mathrm{~g} / \mathrm{L}$ glycerol and $0.4 \%$ glucose were inoculated with fresh transformants and grown overnight at $30^{\circ} \mathrm{C}$ at $175 \mathrm{RPM}$. The overnight cultures were diluted into $3 \mathrm{~L}$ (RTX) or $1.5 \mathrm{~L}$ (RTX ${ }^{\text {exo-}}$ ) fresh TB medium supplemented with $8 \mathrm{~g} / \mathrm{L}$ glycerol and carbenicillin $(50 \mu \mathrm{g} / \mathrm{mL})$. The cultures were grown at $37^{\circ} \mathrm{C}$ in the LEX system and the $\mathrm{OD}_{600}$ was measured periodically until $\mathrm{OD}_{600} 2$ at which the temperature was set to $18^{\circ} \mathrm{C}$. Protein expression was induced at approximately $\mathrm{OD}_{600} 3$ with $1 \mathrm{mM}$ (RTX) or $0.5 \mathrm{mM}$ (RTX ${ }^{\text {exo-}}$ ) IPTG and the cultures were grown additionally for $24 \mathrm{~h}$. The cells were sedimented by centrifugation at $4500 \times g$ 
for $10 \mathrm{~min}$ at $4{ }^{\circ} \mathrm{C}$. The cell pellets were then resuspended in RTX IMAC lysis buffer (100 mM HEPES, $500 \mathrm{mM}$ $\mathrm{NaCl}, 10 \%$ glycerol, $10 \mathrm{mM}$ imidazole, $0.5 \mathrm{mM}$ TCEP, $\mathrm{pH} 8.0$ ) and stored at $-80^{\circ} \mathrm{C}$.

The resuspended cells from each culture were thawed at room temperature and subsequently lysed by pulsed sonication (4 s/4 s $4 \mathrm{~min}, 80 \%$ amplitude, Sonics Vibracell-VCX750, Sonics \& Materials Inc., Newtown, CT, US). The crude cell lysates were centrifuged for $20 \mathrm{~min}$ at $49,000 \times \mathrm{g}$ at $4{ }^{\circ} \mathrm{C}$. For RTX and RTX $\mathrm{X}^{\text {exo- }}$ the supernatants were incubated for $20 \mathrm{~min}$ at $65^{\circ} \mathrm{C}$ and subsequently clarified by centrifugation at $49,000 \times \mathrm{g}$ for $20 \mathrm{~min}$ at $4{ }^{\circ} \mathrm{C}$. The supernatants were filtered through a $0.45 \mu \mathrm{m}$ filter (Corning bottle-top vacuum filter, $0.45 \mu \mathrm{m}$, Corning, NY, USA), and then loaded onto a pre-equilibrated $1 \mathrm{~mL}$ (MashUp-RT) or $5 \mathrm{~mL}$ (RTX and RTX ${ }^{\text {exo-}}$ ) HisTrap HP column (Cytiva, Little Chalfont, UK). All proteins were purified using ÄKTAXpress systems (Cytiva, Little Chalfont, UK). For MashUp-RT, the IMAC column was washed with 20 column volumes (CV) of MashUp-RT IMAC lysis buffer $(25 \mathrm{mM}$ Tris- $\mathrm{HCl}, 300 \mathrm{mM} \mathrm{NaCl}, 10 \%$ glycerol, $40 \mathrm{mM}$ imidazole, $0.5 \%$ Triton X-100, $\mathrm{pH}$ 8 ) and the bound protein was eluted with $2 \mathrm{CV}$ of $0-6 \%$ MashUp-RT IMAC elution buffer (25 mM Tris- $\mathrm{HCl}$, $300 \mathrm{mM} \mathrm{NaCl}, 10 \%$ glycerol, $500 \mathrm{mM}$ imidazole, $0.5 \%$ Triton X-100, pH 8), 2 CV of $6-10 \%$ elution buffer and finally $24 \mathrm{CV}$ of $10-100 \%$ elution buffer. For RTX and RTX ${ }^{\text {exo-- }}$, the IMAC column was washed with $20 \mathrm{CV}$ of wash buffer 1 (20 mM HEPES, $500 \mathrm{mM} \mathrm{NaCl}, 10 \%$ glycerol, $10 \mathrm{mM}$ imidazole, $0.5 \mathrm{mM}$ TCEP, pH 7.5) and 20 CV of wash buffer 2 (20 mM HEPES, $500 \mathrm{mM} \mathrm{NaCl}, 10 \%$ glycerol, $50 \mathrm{mM}$ imidazole, $0.5 \mathrm{mM}$ TCEP, pH 7.5) and thereafter eluted with $5 \mathrm{CV}$ of RTX IMAC elution buffer $(20 \mathrm{mM}$ HEPES, $500 \mathrm{mM} \mathrm{NaCl}, 10 \%$ glycerol, $500 \mathrm{mM}$ imidazole, $0.5 \mathrm{mM}$ TCEP, pH 7.5). For RTX and RTX ${ }^{\text {exo- }}$ the proteins were further purified on a HiLoad 16/60 Superdex 200 preparative grade column (Cytiva, Little Chalfont, UK) equilibrated with gel filtration buffer (20 mM HEPES, $300 \mathrm{mM} \mathrm{NaCl}, 10 \%$ glycerol, $0.5 \mathrm{mM}$ TCEP, pH 7.5). Elution fractions were analyzed by SDSPAGE before pooling and the fractions containing the target proteins were concentrated with Amicon Ultra-15 concentration filter units ( $50 \mathrm{kDa}$ cut off, Millipore, Burlington, MA, USA) at $5000 \times g, 4{ }^{\circ} \mathrm{C}$. For MashUp-RT, the elution buffer was exchanged to buffer A (50 mM Tris, $150 \mathrm{mM} \mathrm{NaCl}, 1 \mathrm{mM}$ DTT, $1 \mathrm{mM}$ EDTA, $0.01 \%$ Triton $\mathrm{X}-100, \mathrm{pH}$ 7.5) on a PD-10 column (Cytiva, Little Chalfont, UK) and the purified MashUp-RT was then diluted in buffer B (50 mM Tris, $150 \mathrm{mM} \mathrm{NaCl}, 1 \mathrm{mM}$ DTT, $1 \mathrm{mM}$ EDTA, $0.01 \%$ Triton X-100, 60\% glycerol, pH 7.5) to obtain the MashUp-RT storage buffer (50 mM Tris, $150 \mathrm{mM} \mathrm{NaCl,} 1 \mathrm{mM} \mathrm{DTT,} 1 \mathrm{mM}$ EDTA, 0.01\% Triton X-100, $50 \%$ glycerol, pH 7.5). For RTX and RTX ${ }^{\text {exo- }}$, the elution buffer was exchanged to buffer C (50 mM Tris- $\mathrm{HCl}$, $50 \mathrm{mM} \mathrm{KCl}, 0.1 \%$ Tween-20, $10 \%$ glycerol, $\mathrm{pH}$ 8.0) on a PD-10 column (Cytiva, Little Chalfont, UK) and the purified protein was then diluted in buffer D (50 mM Tris- $\mathrm{HCl}, 50 \mathrm{mM} \mathrm{KCl}, 0.1 \%$ Tween-20, 60\% glycerol, $\mathrm{pH}$ 8.0) to obtain the RTX storage buffer $(50 \mathrm{mM}$ Tris- $\mathrm{HCl}, 50 \mathrm{mM} \mathrm{KCl}, 0.1 \%$ Tween-20, $50 \%$ glycerol, pH 8.0). The protein samples were flash-frozen in liquid nitrogen and stored at $-80^{\circ} \mathrm{C}$. Working aliquots were stored at $-20^{\circ} \mathrm{C}$.

RT-LAMP primer design and positive control. We used either the iLACO ${ }^{8}$ or $A s 1 \mathrm{e}^{12}$ primers as previously described.

The iLACO primers are: F3 (CCACTAGAGGAGCTACTGTA), B3 (TGACAAGCTACAACACGT), FIP (AGG TGAGGGTTTTCTACATCACTATATTGGAACAAGCAAATTCTATGG), BIP (ATGGGTTGGGATTATCCT AAATGTGTGCGAGCAAGAACAAGTG), LF (CAGTTTTTAACATGTTGTGCCAACC) and LB (TAGAGC CATGCCTAACATGCT). The As1e primers are: As1_F3 (CGGTGGACAAATTGTCAC), As1_B3 (CTTCTC TGGATTTAACACACTT), As1_LF (TTACAAGCTTAAAGAATGTCTGAACACT), As1_LB (TTGAATTTA GGTGAAACATTTGTCACG), Ass__FIP (TCAGCACACAAAGCCAAAAATTTATCTGTGCAAAGGAAATTA AGGAG), As1_BIP (TATTGGTGGAGCTAAACTTAAAGCCCTGTACAATCCCTTTGAGTG), As1e_FIP (TCA GCACACAAAGCCAAAAATTTATTTTTCTGTGCAAAGGAAATTAAGGAG) and As1e_BIP (TATTGGTGG AGCTAAACTTAAAGCCTTTTCTGTACAATCCCTTTGAGTG).

All oligonucleotides were purchased from Thermo Fisher Scientific (Waltham, MA, USA) with standard desalting and dissolved in nuclease free water upon arrival.

We generated synthetic fragments of SARS-CoV-2 RNA (200-300 bp) by in vitro transcription of PCR fragments containing part of SARS-CoV-2 sequence and a T7 promoter. For iLACO we amplified the PCR product from the viral genome using T7_FW_iLACO (TAATACGACTCACTATAGGGTCAATAGCCGCCACTAGA) and RV_iLACO (AGAAACGGTGTGACAAGCTAC). For As1/As1e we used T7-HMS1_FW (TAATACGAC TCACTATAGGGTGCTTGTGAAATTGTCGGTGGA) and HMS1_rv (GCTTTTAGAGGCATGAGTAGGC). RNA was produced using the TranscriptAid T7 High Yield Transcription Kit (Thermo Fisher Scientific, Waltham, MA, USA), DNase-treated using the TURBO DNA-free Kit (Thermo Fisher Scientific, Waltham, MA, USA), purified using Ampure XP beads (Beckman Coulter, Brea, CA, USA) and quantified using Qubit RNA HS Assay kit (Thermo Fisher Scientific, Waltham, MA, USA).

RT-LAMP reaction conditions. RT-LAMP assays were assembled in a total reaction volume of 10 (for optimizations and benchmarking) or 20 (for patient sample tests) $\mu \mathrm{l}$ in MicroAmp Fast Optical 96 well reaction plates (Thermo Fisher Scientific, Waltham, MA, USA) on ice. For in-house produced enzymes, each $10 \mu \mathrm{l}$ reaction consisted of: $1 \mu \mathrm{l}$ of 10X LAMP primer mix $(2 \mu \mathrm{M} \mathrm{F}, 2 \mu \mathrm{M}$ B3, $16 \mu \mathrm{M}$ FIP, $16 \mu \mathrm{M}$ BIP, $4 \mu \mathrm{M}$ LF and $4 \mu \mathrm{M} \mathrm{LB}$ ), $1 \mu \mathrm{l}$ of $10 \mathrm{X}$ buffer (one of ThermoPol Reaction Buffer, Isothermal Amplification Buffer or II (New England Biolabs, Ipswich, MA, USA), $0.5 \mu \mathrm{l}$ 20X fluorescent dye (either Eva Green (Biotium, Fremont, CA, USA) or SYBR Green I (Thermo Fisher Scientific, Waltham, MA, USA), $0.6 \mu 100 \mathrm{mM} \mathrm{MgSO}_{4}$ (New England Biolabs, Ipswich, MA, USA), $1.4 \mu \mathrm{l}$ of dNTP mix (10 mM each, New England Biolabs, Ipswich, MA, USA), $0.5 \mu \mathrm{l}$ of strand-displacing polymerase, $0.5 \mu \mathrm{l}$ of in-house reverse transcriptase or $0.2 \mu \mathrm{l}$ SuperScript IV (SSIV, Thermo Fisher Scientific, Waltham, MA, USA), $1 \mu \mathrm{l}$ sample, and nuclease free water (Thermo Fisher Scientific, Waltham, MA, USA) up to $10 \mu$ l. The in-house enzymes were pre-diluted to desired concentration in a buffer containing $10 \mathrm{mM}$ Tris- $\mathrm{HCl}(\mathrm{pH} 7.5)$ and $25 \mathrm{mM} \mathrm{KCl}$. For $20 \mu \mathrm{l}$ reactions, all the volumes above were doubled. 
1X ThermoPol Reaction Buffer contains $20 \mathrm{mM}$ Tris- $\mathrm{HCl}, 10 \mathrm{mM}\left(\mathrm{NH}_{4}\right)_{2} \mathrm{SO}_{4}, 10 \mathrm{mM} \mathrm{KCl}, 2 \mathrm{mM} \mathrm{MgSO}$, $0.1 \%$ Triton X-100, $\mathrm{pH}$ 8.8. $1 \mathrm{X}$ Isothermal Amplification Buffer contains $20 \mathrm{mM}$ Tris- $\mathrm{HCl}, 10 \mathrm{mM}\left(\mathrm{NH}_{4}\right)_{2} \mathrm{SO}_{4}$, $50 \mathrm{mM} \mathrm{KCl}, 2 \mathrm{mM} \mathrm{MgSO}_{4}, 0.1 \%$ Tween 20, pH 8.8, while 1X Isothermal Amplification Buffer II has the same components but more $\mathrm{KCl}(150 \mathrm{mM})$. Some experiments were supplemented with additional $\mathrm{KCl}$ to test the full range of concentrations.

Reactions containing Bst 3.0 DNA Polymerase (New England Biolabs, Ipswich, MA, USA) and Saphir Bst2.0 DNA polymerase (Jena Bioscience, Jena, Germany) were largely performed as described for the in-house reagents. Isothermal amplification buffer II was used with Bst3.0 and Saphir Bst2.0 Turbo Buffer (Jena Bioscience, Jena, Germany) was used with Saphir Bst2.0, as recommended by manufacturers. Enzyme amounts were used as recommended by manufacturers. When using WarmStart Colorimetric RT-LAMP 2X Master Mix (New England Biolabs, Ipswich, MA, USA), $5 \mu \mathrm{lmix}$ was used together with $1 \mu \mathrm{l} 10 \mathrm{X}$ LAMP primer mix, $0.5 \mu \mathrm{l}$ Eva Green dye, $1 \mu \mathrm{l}$ of sample, and nuclease-free water to a final volume of $10 \mu \mathrm{l}$ (double the volumes for $20 \mu \mathrm{l}$ reactions). All reactions were run in a Step One Plus Real time PCR system (Applied Biosystems).

To test the RT activity of RTX and RTX ${ }^{\text {exo-}}$, we performed RT-qPCR according to the following protocol. $10 \mu \mathrm{l}$ R reactions were set up for each condition containing: either ThermoPol buffer, Isothermal Amplification buffer, or Isothermal Amplification buffer II; $1.4 \mathrm{mM}$ each dNTP; $6 \mathrm{mM} \mathrm{MgSO}_{4}$ (where appropriate); $0.2 \mu \mathrm{M}$ iLACO-qPCR-rv primer (AGCATGTTAGGCATGGCTCT); specified amount of either RTX or RTX ${ }^{\text {exo-}}$, or 4 U/ $\mu$ l Superscript IV (SSIV), or no enzyme; 83 million copies of iLACO synthetic RNA template; and nucleasefree water up to $10 \mu \mathrm{l}$. RT was performed at $65^{\circ} \mathrm{C}$ for $15 \mathrm{~min} .1 \mu \mathrm{l}$ of each RT reaction was used as template in a $10 \mu \mathrm{l}$ qPCR reaction using $5 \mu \mathrm{l}$ Fast SYBR Green Master Mix (Thermo Fisher Scientific, Waltham, MA, USA) and $0.5 \mu \mathrm{M}$ each of iLACO_qPCR_fw (TATGGTGGTTGGCACAACAT) and iLACO_qPCR_rv primers. qPCR was performed according to the standard protocol.

Numeric raw data for the amplification curves is provided in Table S1.

Clinical samples. We obtained 184 nasopharyngeal samples from Karolinska University Hospital, Huddinge (Stockholm) collected between May 20th and June 1st 2020. We used pseudo-anonymized surplus material previously collected for clinical diagnostics of SARS-CoV-2. This is in accordance with the Swedish Act concerning the Ethical Review of Research Involving Humans, which allows development and improvement of diagnostic assays using patient samples which were collected to perform the testing in question. Thus, according to Swedish law neither specific ethical approval nor informed consent was required. Pseudo-anonymized means that data was process in such a way that it can no longer be attributed to a specific subject without the use of additional information, and the additional information is separate from the authors, making it virtually impossible to identify any individual. We picked samples collected in either Sigma-Transwab, Sigma-Virocult (MWE, Corsham, Wiltshire, England) or COPAN-eSwab (Copan Diagnostics Inc., CA, USA) kits. All samples were first analyzed on the GeneXpert Xpress SARS-CoV-2 detection system (Cepheid, Sunnyvale, CA, USA) at the hospital and called as either positive (142 samples) or negative (42 samples) (Table S2). Samples which had at least a determined GeneXpert Ct value for N2 were called as positive, while samples where both GeneXpert Ct values (N2 and E) were not determined were called as negative (samples where only E had a Ct value were re-tested). We therefore used the N2 Ct values for comparison with our RT-LAMP results.

For RT-LAMP, $50 \mu \mathrm{l}$ of each sample was heat-inactivated at $95{ }^{\circ} \mathrm{C}$ for $15 \mathrm{~min}$ and stored at $-80{ }^{\circ} \mathrm{C}$ prior to experiments. Two $\mu \mathrm{l}$ of unextracted sample were used in $20 \mu \mathrm{l}$ total reaction volume.

Ethics statement. We used pseudo-anonymized surplus material previously collected for clinical diagnostics of SARS-CoV-2. This is in accordance with the Swedish Act concerning the Ethical Review of Research Involving Humans, which allows development and improvement of diagnostic assays using patient samples which were collected to perform the testing in question. Pseudo-anonymized means that data was process in such a way that it can no longer be attributed to a specific subject without the use of additional information, and the additional information is separate from the authors making virtually impossible to identify any individual. Additional ethical approval for RT-LAMP diagnosis was obtained by the appropriate Swedish Authority (Dnr 2020-01945, ethics committee: Etikprövningsnämnden).

\section{Results}

Purification and optimization of alternative LAMP enzymes. As a starting point to optimize alternative reagents for LAMP testing, we used the thermophilic strand-displacing polymerases developed by the Ellington Lab ${ }^{20}$. We used both Bst LF from Geobacillus stearothermophilus as well as the chimeras v5.9 and v7.16 of Bst LF and Klentaq (Thermus aquaticus) which displayed higher thermal stability ${ }^{20}$. We performed simple enzyme expression and purification to enable effortless production of good quality reagents for hundreds of thousands of tests (see methods). To validate the performance of the synthesized enzymes in SARS-CoV-2 RTLAMP detection, we first benchmarked their ability to amplify in vitro produced SARS-CoV-2 RNA fragments using the iLACO primer set that we previously developed ${ }^{8}$ (Fig. 1 and Table S1).

We tested the intrinsic reverse transcriptase (RT) activity of all enzymes, and compared their ability to efficiently amplify and detect the synthetic RNA fragment with or without supplementing the reaction with a commercial thermostable RT enzyme (Superscript IV, SSIV). Addition of a thermostable RT was essential to enhance the performance of all tested enzymes (not shown), thus, we carried out further optimizations in the presence of SSIV. To facilitate quantitative comparison between samples, we performed all the optimizations using continuous fluorescence detection. However, to increase throughput and avoid the use of expensive equipment, end-point measurement of fluorescence or colorimetric detection can also be applied ${ }^{8-13,17,21}$. 


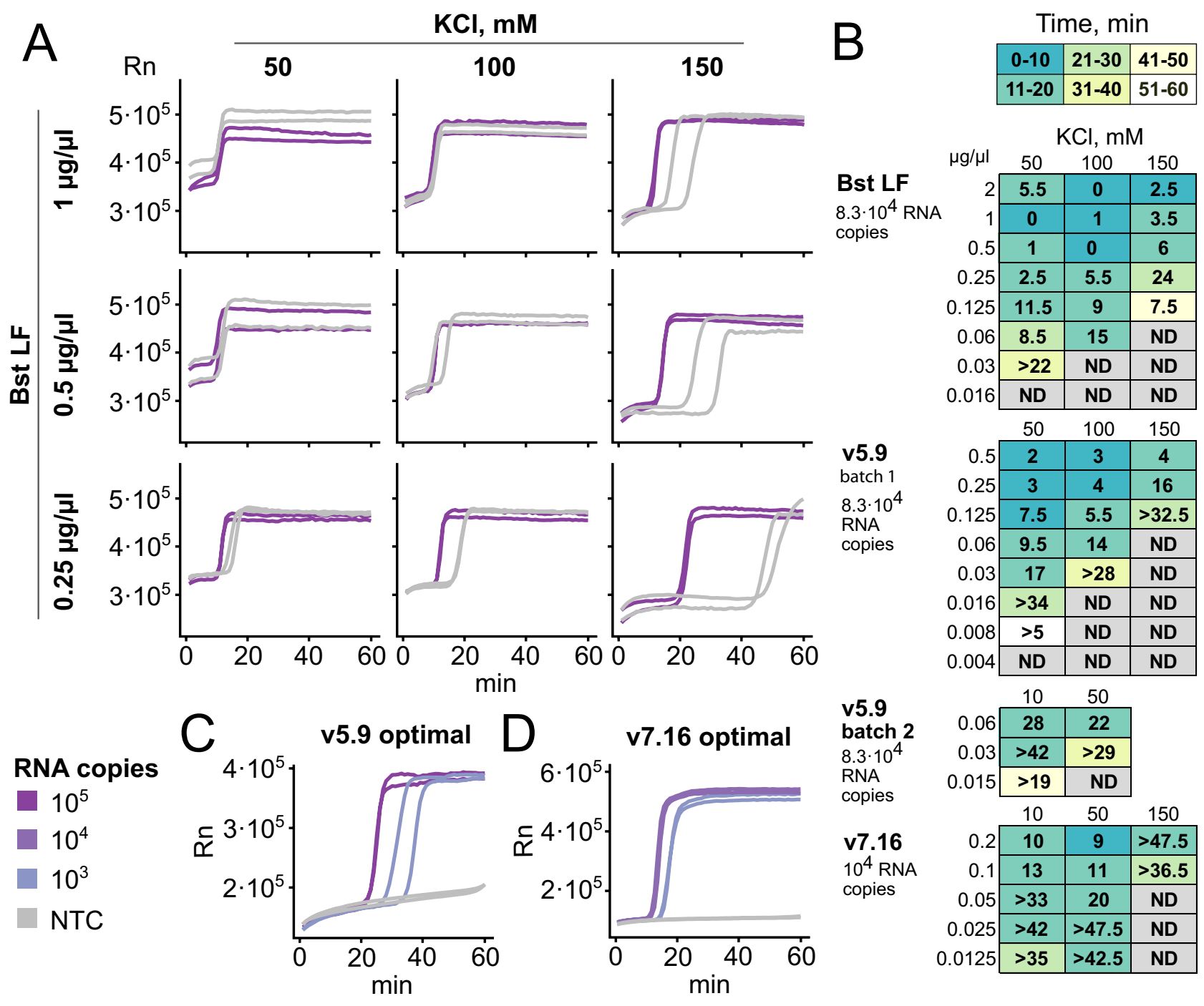

Figure 1. Optimization of enzyme amount and buffer composition for RT-LAMP. All experiments shown were set up with iLACO primers and run for $1 \mathrm{~h}$ at $65^{\circ} \mathrm{C}$ in a thermocycler, tracked by either SYBR Green I (Bst LF, v5.9) or Eva Green (v7.16) fluorescence. (A) Example of RT-LAMP optimization varying Bst LF amount and the $\mathrm{KCl}$ concentration. Positive control synthetic RNA (83,000 copies) and no template control (each in duplicate) were assayed. (B) Summary of optimization of enzyme amount and $\mathrm{KCl}$ concentration for Bst LF, v5.9, and v.7.16. Numbers indicate the time difference between the slowest-amplifying positive control replicate and the fastest-amplifying negative control replicate. Color indicates the required time to detect the slowest-amplifying positive control. See Fig. S1 for a additional details regarding how tables are contructed. " $>$..." indicates that the negative control did not amplify within $1 \mathrm{~h}$. ND indicates no amplification of either positive or negative control. Two batches of v5.9 with differing activity were assayed, and batch 2 was used for further experiments. (C-D) Optimal conditions determined for v5.9 $(0.03 \mu \mathrm{g} / \mu \mathrm{l}$ in ThermoPol buffer, $10 \mathrm{mM} \mathrm{KCl})$ and v7.16 $(0.025 \mu \mathrm{g} / \mu \mathrm{l}$ in isothermal amplification buffer, $50 \mathrm{mM} \mathrm{KCl}$ ). 83,000 and 830 copies of RNA were used with v5.9, 10,000 and 1000 with v7.16.

We optimized the composition of the reaction buffer and the amount of strand displacing DNA polymerase used (Fig. 1A,B). For a fixed template amount we minimized the time to detection (color in Fig. 1B) and maximized the time between true positive and false positive (spurious amplification, number in Fig. 1B; see Fig. S1 for detail). Non-specific spurious LAMP amplification is a known phenomenon that can lead to false positive detection and is templated by the primers themselves ${ }^{22}$. In some cases, the amplification of spurious non-specific sequences can be detected by performing a melting curve analysis (Fig. S2) ${ }^{23}$. However, to avoid false positives, reaction conditions and components should be optimized to maximize sensitivity while minimizing spurious amplification. Variation of any component of the reaction (e.g., oligos, enzyme, buffer composition...) as well as the physical reaction conditions (e.g., temperature, time...) can affect the spurious amplification. For example, while increased enzyme concentration decreases time to detection, it also increases false positive amplification (Fig. 1B,C). We observed that the optimal concentration of $\mathrm{KCl}$ varied between the enzymes tested. $150 \mathrm{mM}$ $\mathrm{KCl}$ was optimal for Bst LF, $50 \mathrm{mM}$ for v7.16, and $10 \mathrm{mM}$ for v5.9. Based on previous literature, we tested the 


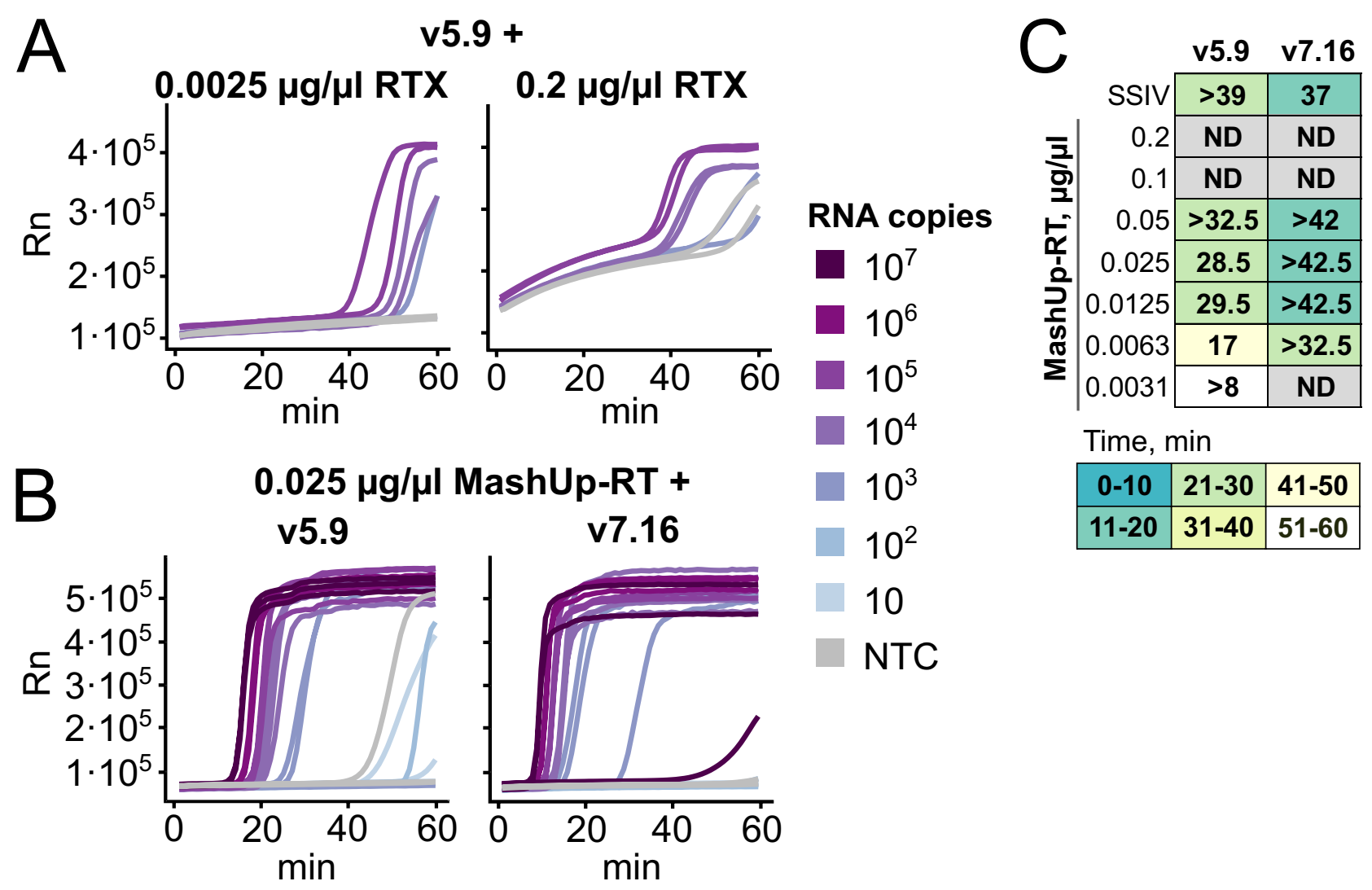

Figure 2. Compatibility of v5.9 and v7.16 polymerases with non-commercial thermostable RT enzymes. All experiments shown were set up with iLACO primers and run for $1 \mathrm{~h}$ at $65^{\circ} \mathrm{C}$ in a thermocycler, tracked by Eva Green fluorescence. (A) Example amplification plots showing the performance of v5.9 with low or high amounts of RTX (duplicates of 83,000, 8300, and 830 copies of synthetic RNA as well as non template control (NTC)). (B) Optimal detection of synthetic SARS-CoV-2 RNA using v5.9 $(0.03 \mu \mathrm{g} / \mu \mathrm{l}$ in ThermoPol buffer $)$ or v7.16 $(0.025 \mu \mathrm{g} / \mu \mathrm{l}$ in IA buffer) supplemented with MashUp-RT as the thermostable reverse transcriptase. Reactions were performed in triplicate. (C) Optimization of MashUp-RT enzyme amount in combination with v5.9 and v7.16 in their respective optimal conditions (see above for B). 10,000 copies of synthetic RNA and NTC were assayed. Time between positive and negative amplification is indicated in minutes and the color indicates time for detection of the positive controls, as in Fig. 1B.

capacity of betaine $\mathrm{e}^{24}$, guanidine $\mathrm{e}^{25}$ and $\mathrm{DMSO}^{26}$ to increase sensitivity and reduce nonspecific amplification. Unfortunately, in our hands none of these strategies improved sensitivity or specificity. It is important to note that even the dye used for fluorescent detection can alter the measured RT-LAMP sensitivity and specificity and thus should also be accounted for in optimization. In our conditions, Eva Green dye provided a higher dynamic range than SYBR Green I, when measuring difference in fluorescence before and after RT-LAMP reaction. We compared the three polymerases based on the speed of amplification, the specificity, and the range of conditions where the former two parameters are acceptable. Specifically, we selected the minimal amount of enzyme capable of amplifying $10^{5}$ copies of RNA template before $20 \mathrm{~min}$ with no measurable amplification of the non-template control before $50 \mathrm{~min}$. We found that v7.16 outperformed v5.9, and both outperformed Bst LF. Amplification curves for LAMP at optimal conditions with v5.9 and v7.16 are shown in Figs. 1C,D (raw data in Table S2). We proceeded with both v5.9 and v7.16 in the following experiments.

Optimization of alternative RT enzymes compatible with LAMP. Once we optimized the conditions for efficient strand-displacement DNA activity required for LAMP, we decided to explore alternatives to the commercial thermostable RT enzymes. First, we explored the use of a thermostable synthetic reverse transcriptase, RTX, developed by the Ellington $l \mathrm{ab}^{27}$, which can also be used for direct RT-qPCR of SARS-CoV-2 $2^{18}$. However, supplementing either v5.9 or v7.16 with different concentrations of RTX did not improve their ability to detect SARS-CoV-2 RNA (Fig. 2A and Table S1). Addition of high concentrations of RTX lead to abnormal amplification curves, while lower concentrations did not improve the RT-LAMP activity of v5.9 and v7.16. Similar results were obtained using both the RTX versions containing or excluding the proofreading domain (Fig. S3A-D). Importantly, the absence of improvement in the LAMP reaction was not due to lack of RT activity of these enzymes, as demonstrated by standard RT-qPCR (Fig. S3E). Thus, we concluded that RTX is not compatible with RT-LAMP.

In addition to RTX, we aimed to explore other non-commercial alternatives. MashUp-RT is based on FeLV$\mathrm{RT}$, which is intrinsically more accurate than MMLV-RT ${ }^{28}$ and contains mutations which further increase 


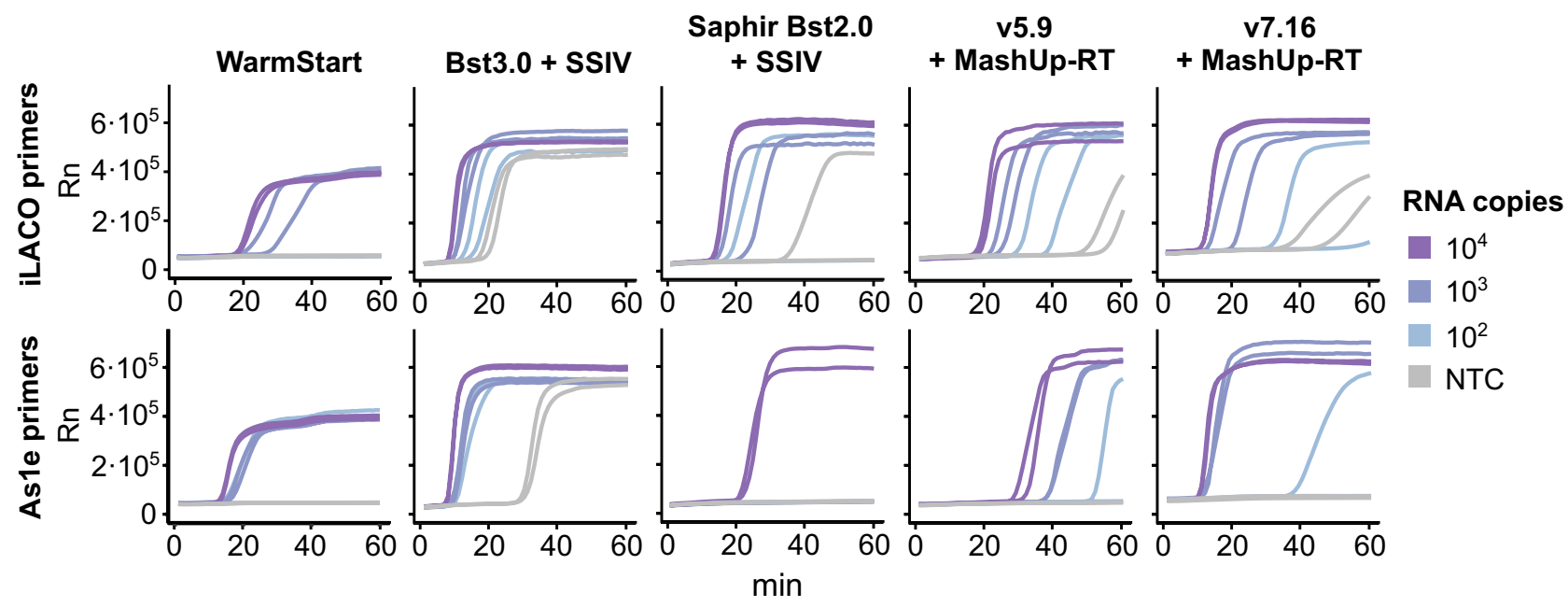

Figure 3. Benchmarking of in-house produced enzymes against commercial alternatives. Synthetic RNA templates (iLACO and As1e) amplified with the corresponding primers and either WarmStart, Bst3.0 with SSIV, Saphir Bst2.0 Turbo with SSIV, v5.9 with MashUp-RT, or v7.16 with MashUp-RT (same conditions for v5.9 and v7.16 as in Fig. 2B). All experiments were run for $1 \mathrm{~h}$ at $65^{\circ} \mathrm{C}$ in a thermocycler, tracked by Eva Green fluorescence.

thermostability and fidelity (https://pipettejockey.com/). We expressed MashUp-RT and tested its ability to enhance RT-LAMP reaction in combination with v5.9 and v7.16. The addition of $0.025 \mu \mathrm{g} / \mu \mathrm{l}$ reaction was enough for a performance identical to, or even better than, that of SSIV (Fig. 2B,C). This shows that MashUp-RT is a thermostable RT enzyme compatible with LAMP that is capable of enhancing RT-LAMP detection sensitivity for SARS-CoV-2 RNA.

Benchmarking of alternative RT-LAMP enzyme mix and oligo combinations. Once we optimized non-commercial enzyme RT-LAMP conditions, we benchmarked their ability to detect SARS-CoV-2 against commercial alternatives. After testing multiple oligo primer sets optimized for SARS-CoV-2 $2^{8,10,12}$, we decided to focus on the use of iLACO ${ }^{8}$ and As1 $\mathrm{e}^{12}$ targeting ORF1ab, which worked better in our hands. We compared our optimized enzyme mix with commercial alternatives from multiple providers (i.e., WarmStart Colorimetric master mix (NEB), Bst3.0 (NEB) and Saphir Bst2.0 Turbo (Jena Biosciences)) using synthetic RNA (Fig. 3 and Table S1). Although all enzymes were able to detect SARS-CoV-2 RNA, we identified clear differences in both sensitivity and background level. In particular, the performance of the RT-LAMP reaction using v7.16 enzyme and Asle primers was comparable to the best commercial alternatives, with a detection speed similar to Bst3.0 and lower spurious amplification. Unexpectedly, we found that Bst 3.0 needed to be supplemented with RT activity to detect SARS-CoV-2 RNA (Fig. S4). The performance of primer sets was also affected by the different enzymes used. For example, v5.9 and Saphir Bst2.0 perform better in combination with iLACO primers, while other enzymes perform better with Asle or similarly with both. Thus, we concluded that in-house produced v5.9 or v7.16 in combination with MashUp-RT and iLACO or Asle primers were competitive alternatives for RT-LAMP detection of purified RNA.

Application of optimized RT-LAMP to clinical nasopharyngeal samples. Since we and others have already shown the utility of RT-LAMP for the detection of SARS-CoV-2 from patient purified RNA ${ }^{8-11,17}$, we now wanted to explore the ability of our in-house enzyme mix to detect SARS-CoV-2 also in non-purified samples ${ }^{11,17}$.

We first explored its compatibility with different virus transport media commonly used for the collection of nasopharyngeal swabs: Virocult (MWE), Sigma Transwab (MWE), eSwab (Copan) and Beaver (BEAVER biomedical) (Fig. 4A and Table S1). Three of the four tested media (Virocult, Transwab and eSwab) were compatible with both v7.16 and v5.9 reactions when adding $10 \%$ of the reaction volume. However, the Beaver media required additional dilution (three-fold) to avoid inhibition and was compatible only with v5.9. This limits the total amount of patient sample that could be used per reaction and thus decreases the overall sensitivity.

Having established the reaction conditions, we used 184 nasopharyngeal patient samples collected in Virocult, TransSwab and eSwab transport media that have been previously analyzed using GeneXpert SARS-CoV-2 detection (Cepheid) at the Karolinska University Hospital (Sweden). We selected 142 positive samples across all GeneXpert $\mathrm{Ct}$ ranges, and 42 samples determined to be negative for SARS-CoV-2. We used our developed v7.16 + MashUp-RT reaction mix in combination with either iLACO or Asle primer sets (Fig. 4B,D and Table S2). While both primer sets were successful in detecting samples with high viral load, the Asle primers showed better overall sensitivity. Performing technical duplicates demonstrated that the sensitivity was further increased if a sample was called as positive when at least one replicate had amplified (Fig. 4C). Unfortunately, in all cases patient samples with low-to-medium content of SARS-CoV-2 RNA (higher GeneXpert Cts) were often misclassified as false negatives. Finally, we compare the results obtained with our in-house reagents with state-of-the-art 
A
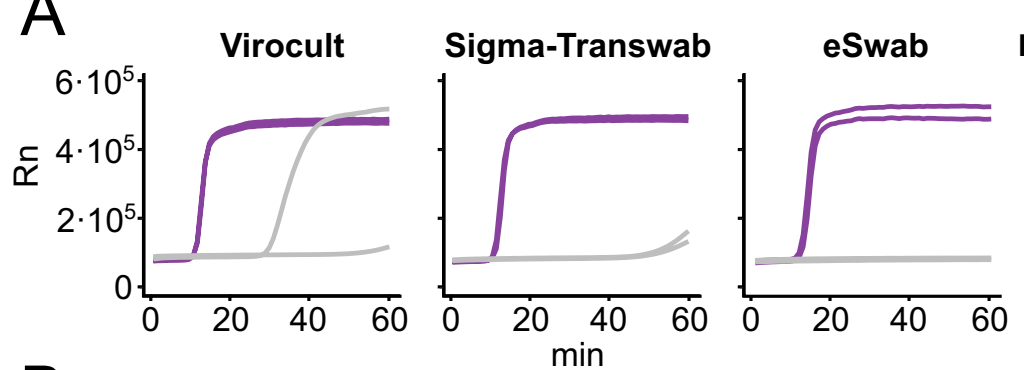

RNA copies

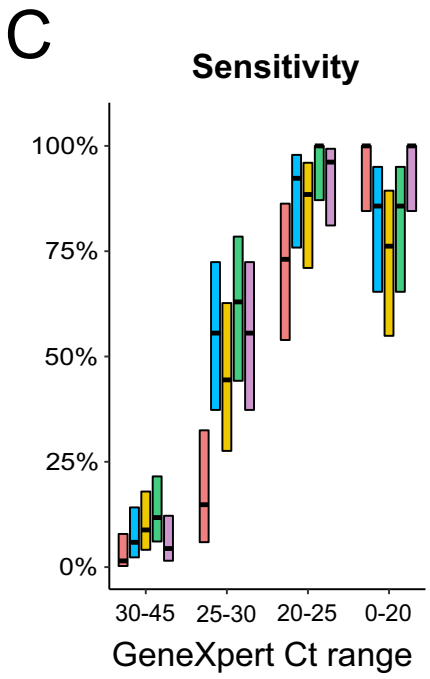

\section{B WarmStart}

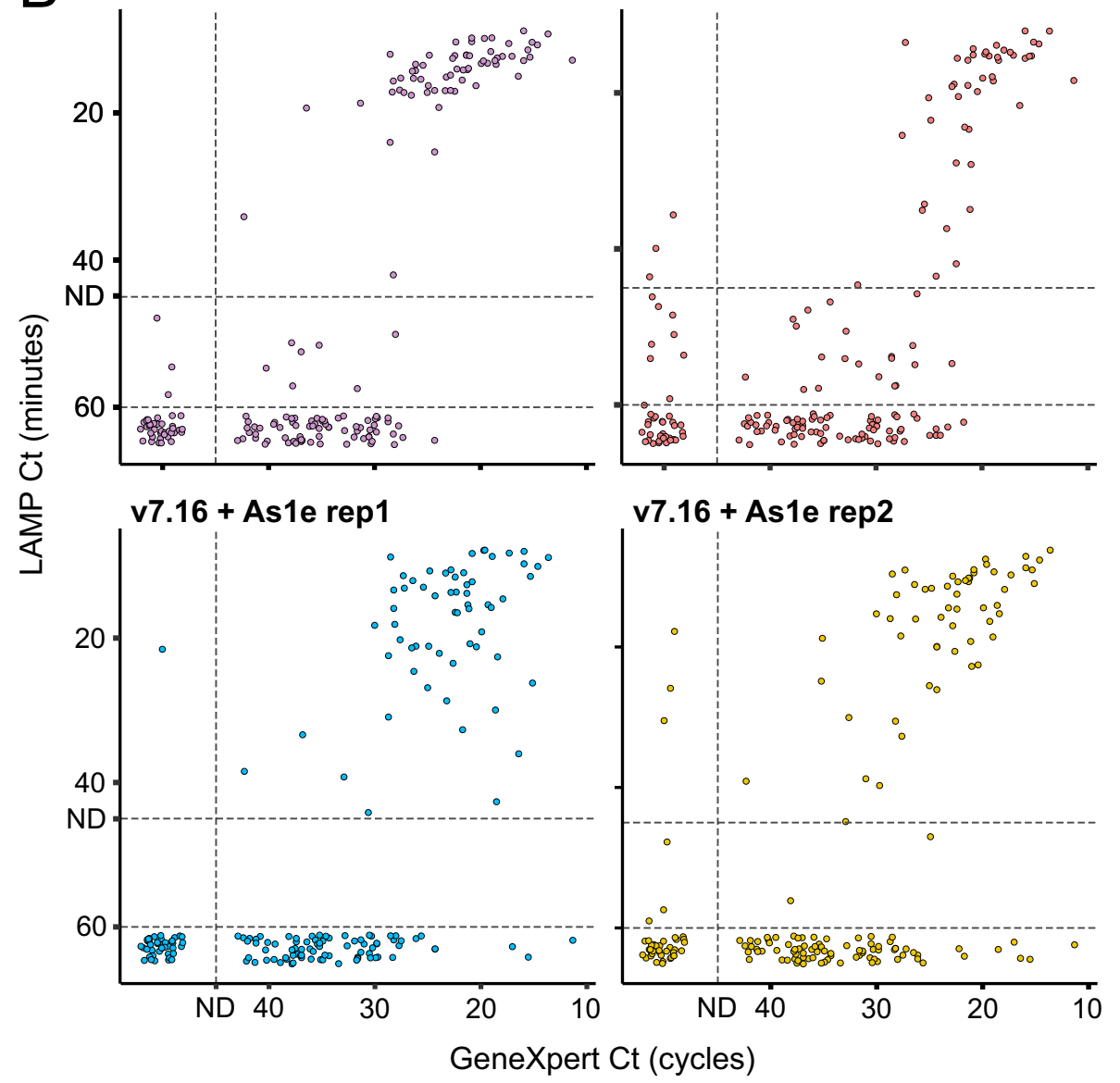

v7.16 + iLACO

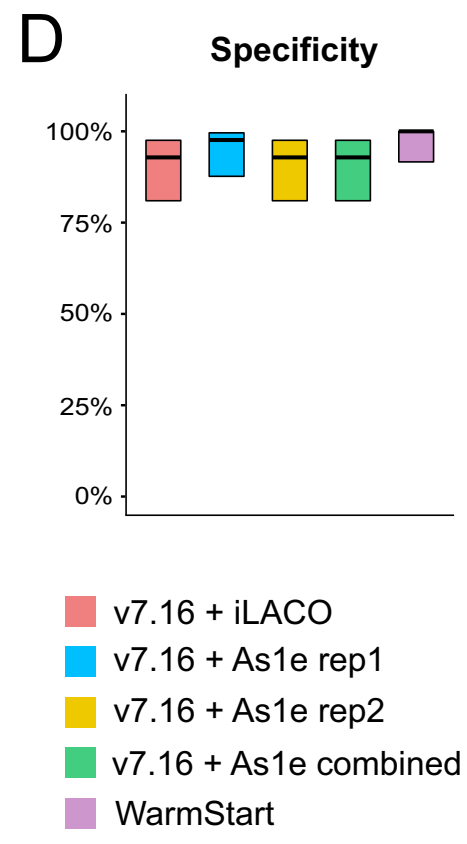

Figure 4. Applicability of RT-LAMP to unextracted nasopharyngeal samples. (A) Effect of common virus transport media on RT-LAMP amplification with v7.16 and MashUp-RT (same conditions and same experiment as in Fig. 2B). Different transport media were added at $10 \%$ of the reaction volume. (B) Comparisons of RT-LAMP Ct (minutes) and GeneXpert RT-qPCR Ct (cycles) for 184 clinical samples. The developed v7.16 + RT-MashUP reaction mix was tested with iLACO (red) or As1e primer sets (blue/yellow). Asle primer set was also tested with commercial WarmStart Colorimetric master mix (violet). ND designates the thresholds for calling positives (see methods). (C) Reaction sensitivity according to SARS-CoV-2 abundance as determined by GeneXpert. When technical replicates of Asle were performed and considered together, sensitivity improved (in green, positives were called when at least one replicate was identified as positive). Number of samples in each Ct range: Ct $0-20=21$, Ct $20-25=26$, Ct $25-30=27$, Ct 30-45=68. Wilson's binomial confidence intervals of $95 \%$ are shown ${ }^{17}$. (D) Reaction specificity, as $\%$ of samples considered negatives by RT-qPCR that were also negative by RT-LAMP. Wilson's binomial confidence intervals of $95 \%$ are shown.

commercial reagents (Warmstart Colorimetric master mix) and demonstrate that they have comparable performance in terms of specificity and sensitivity. In summary, we have shown how in-house optimized enzyme mixes offer a high-quality economic alternative to current commercial products. 


\section{Discussion}

Here we have optimized and validated the production and application of alternative non-commercial reagents for simplified RT-LAMP based SARS-CoV-2 detection. We demonstrate their ability to identify COVID-19 positive patients using unextracted nasopharyngeal swabs in common virus transport media. And finally, we show that even omitting RNA purification, RT-LAMP can easily detect samples with high to medium SARS-CoV-2 RNA content. This is especially important when considering that frequent testing, even at a lower sensitivity, can help to effectively decrease disease spread ${ }^{1-3}$.

During this optimization process we obtained some general recommendations that can facilitate the application of RT-LAMP in different settings. In addition to the initial benchmarking with alternative RT-qPCR approaches, it is important to routinely control for spurious amplification and cross contamination. For example, it is common to observe variation in the quality and specificity of different oligonucleotide batches (even from the same manufacturer). In some circumstances, oligonucleotide batches can contain a trace amount of target regions from the SARS-CoV-2 genome due to oligo cross contamination during oligonucleotide synthesis or handling. Thus, reactions with negative water controls should always be performed in parallel to ensure the specificity of the test.

The activity of each new batch of enzymes and reagents should first be calibrated with a known serial dilution of target RNA. In particular, we recommend assessing multiple concentrations of the newly produced protein (v5.9 or v7.16) in a range from 0.01 to $1 \mu \mathrm{g} / \mu \mathrm{l}$ in reaction. We propose to estimate batch activity by assaying the minimal amount of enzyme capable of amplifying $10^{5}$ copies of RNA template before 20 min with no measurable amplification of the non-template control before $50 \mathrm{~min}$. If end-point measurement is used instead of real-time tracking, the time at which the reaction is stopped should also be optimized. These parameters should be estimated whenever a new batch of enzyme is produced, which is usually enough for tens of thousands of reactions. However, we suggest to re-assay if loss of activity is suspected.

Although direct RT-LAMP in unextracted samples can detect most cases with medium to high SARS-CoV-2 viral load, to bring it to a RT-qPCR detection sensitivity level it is still necessary to perform at least some simple sample purification and RNA concentration ${ }^{9-11,15}$. In addition, even if the use of viral transport media can be tolerated by RT-LAMP, less complex media, or no media at all, might be more advantageous ${ }^{29}$. Our work also highlights the importance of controlling for the presence of salt and other components that may affect RT-LAMP sensitivity and specificity. In summary, we hope that this work will facilitate the implementation of affordable RT-LAMP SARS-CoV-2 diagnostics in settings where the use of commercial reagents is not possible. This could decrease the total cost per test in order to facilitate routine testing of large portions of the population.

Received: 28 September 2020; Accepted: 20 December 2020

Published online: 19 January 2021

\section{References}

1. Taipale, J., Romer, P. \& Linnarsson, S. Population-scale testing can suppress the spread of COVID-19. Preprint at https://www. medrxiv.org/content/10.1101/2020.04.27.20078329v2medRxiv (2020).

2. Paltiel, A. D., Zheng, A. \& Walensky, R. P. Assessment of SARS-CoV-2 screening strategies to permit the safe reopening of college campuses in the United States. JAMA Netw. Open 3, e2016818 (2020).

3. Larremore, D. B. et al. Test sensitivity is secondary to frequency and turnaround time for COVID-19 surveillance. Preprint at https ://www.medrxiv.org/content/10.1101/2020.06.22.20136309v3 (2020).

4. Esbin, M. N. et al. Overcoming the bottleneck to widespread testing: a rapid review of nucleic acid testing approaches for COVID19 detection. RNA 26, 771-783 (2020).

5. Yan, L. et al. Isothermal amplified detection of DNA and RNA. Mol. Biosyst. 10, 970-1003 (2014).

6. Notomi, T. et al. Loop-mediated isothermal amplification of DNA. Nucleic Acids Res. 28, E63 (2000).

7. Augustine, R. et al. Loop-mediated isothermal amplification (LAMP): a rapid, sensitive, specific, and cost-effective point-of-care test for coronaviruses in the context of COVID-19 pandemic. Biology 9, 182 (2020).

8. Yu, L. et al. Rapid detection of COVID-19 coronavirus using a reverse transcriptional loop-mediated isothermal amplification (RT-LAMP) diagnostic platform. Clin. Chem. 66, 975-977 (2020).

9. Park, G.-S. et al. Development of reverse transcription loop-mediated isothermal amplification assays targeting severe acute respiratory syndrome coronavirus 2 (SARS-CoV-2). J. Mol. Diagn. 22, 729-735 (2020).

10. Zhang, Y. et al. Rapid Molecular Detection of SARS-CoV-2 (COVID-19) Virus RNA Using Colorimetric LAMP. Preprint at https ://www.medrxiv.org/content/10.1101/2020.02.26.20028373v1 (2020).

11. Max J. Kellner et al. A rapid, highly sensitive and open-access SARS-CoV-2 detection assay for laboratory and home testing. Preprint at https://www.biorxiv.org/content/10.1101/2020.06.23.166397v2 (2020).

12. Rabe, B. A. \& Cepko, C. SARS-CoV-2 detection using isothermal amplification and a rapid, inexpensive protocol for sample inactivation and purification. Proc. Nat. Acad. Sci. (PNAS) 117, 24450-24458 (2020).

13. Klein, S. et al. SARS-CoV-2 RNA extraction using magnetic beads for rapid large-scale testing by RT-qPCR and RT-LAMP. Viruses 12, 863 (2020).

14. Joung, J. et al. Point-of-care testing for COVID-19 using SHERLOCK diagnostics. Preprint at https://www.medrxiv.org/conte $\mathrm{nt} / 10.1101 / 2020.05 .04 .20091231 \mathrm{v1}(2020)$.

15. Broughton, J. P. et al. CRISPR-Cas12-based detection of SARS-CoV-2. Nat. Biotechnol. 38, 870-874 (2020).

16. Schmid-Burgk, J. L. et al. LAMP-Seq: Population-Scale COVID-19 Diagnostics Using Combinatorial Barcoding. Preprint at https ://www.biorxiv.org/content/10.1101/2020.04.06.025635v2 (2020).

17. Dao Thi, V. L. et al. A colorimetric RT-LAMP assay and LAMP-sequencing for detecting SARS-CoV-2 RNA in clinical samples. Sci. Transl. Med. 12, eabc7075 (2020).

18. Bhadra, S., Maranhao, A. C. \& Ellington, A. D. A one-enzyme RT-qPCR assay for SARS-CoV-2, and procedures for reagent production. Preprint at https://www.biorxiv.org/content/10.1101/2020.03.29.013342v5 (2020).

19. Bhadra, S., Riedel, T. E., Lakhotia, S., Tran, N. D. \& Ellington, A. D. High-surety isothermal amplification and detection of SARSCoV-2, including with crude enzymes. Preprint at https://www.biorxiv.org/content/10.1101/2020.04.13.039941v3 (2020).

20. Milligan, J. N., Shroff, R., Garry, D. J. \& Ellington, A. D. Evolution of a thermophilic strand-displacing polymerase using hightemperature isothermal compartmentalized self-replication. Biochemistry 57, 4607-4619 (2018). 
21. Tanner, N. A., Zhang, Y. \& Evans, T. C. Visual detection of isothermal nucleic acid amplification using pH-sensitive dyes. Biotechniques 58, 59-68 (2015).

22. Meagher, R. J., Priye, A., Light, Y. K., Huang, C. \& Wang, E. Impact of primer dimers and self-amplifying hairpins on reverse transcription loop-mediated isothermal amplification detection of viral RNA. Analyst 143, 1924-1933 (2018).

23. Rolando, J. C., Jue, E., Barlow, J. T. \& Ismagilov, R. F. Real-time kinetics and high-resolution melt curves in single-molecule digital LAMP to differentiate and study specific and non-specific amplification. Nucleic Acids Res. 48, e42 (2020).

24. Henke, W., Herdel, K., Jung, K., Schnorr, D. \& Loening, S. A. Betaine improves the PCR amplification of GC-rich DNA sequences. Nucleic Acids Res. 25, 3957-3958 (1997).

25. Zhang, Y. et al. Enhancing Colorimetric LAMP Amplification Speed and Sensitivity with Guanidine Chloride. Preprint at https:// www.biorxiv.org/content/10.1101/2020.06.03.132894v1 (2020).

26. Wang, D.-G., Brewster, J. D., Paul, M. \& Tomasula, P. M. Two methods for increased specificity and sensitivity in loop-mediated isothermal amplification. Molecules 20, 6048-6059 (2015).

27. Ellefson, J. W. et al. Synthetic evolutionary origin of a proofreading reverse transcriptase. Science 352, 1590-1593 (2016).

28. Menéndez-Arias, L. Mutation rates and intrinsic fidelity of retroviral reverse transcriptases. Viruses 1, 1137-1165 (2009).

29. Ott, I. M. et al. Simply saliva: stability of SARS-CoV-2 detection negates the need for expensive collection devices. Preprint at https ://www.medrxiv.org/content/10.1101/2020.08.03.20165233v1 (2020).

\section{Acknowledgements}

We thank Andy Ellington and Alexander Klenov (https://pipettejockey.com/) for sharing reagents. We thank Björn Högberg, Sten Linnarsson, Michael Knop, Simon Anders, Uwe Sauer and the whole SciLifeLab community for discussion and support.

\section{Author contributions}

A.A., D.B., and Y.P.S., designed and carried out experiments and performed data analysis. T.N., S.J.E., R.J.H., E.S., H.A.K. and U.R. performed and optimized enzyme production. J.M. and A.K. optimized conditions for RT-Mashup expression. G.S, B.R. and S.Z.V handled clinical samples and advised regarding clinical RT-LAMP optimization. X.Y., S.Y. and X.L. contributed to method optimization for RT-LAMP. X.Y and V.P. conceived the original idea. V.P. wrote the initial manuscript with support from A.A., D.B., and Y.P.S. All authors contributed to the writing of the final manuscript. V.P. supervised the project. All authors approved the final draft.

\section{Funding}

Open Access funding provided by Karolinska Institute. This project was primarily funded by the SciLifeLab/ KAW national COVID-19 research program Project Grant (KAW 2020.0182). VP laboratory is supported by a Wallenberg Academy Fellowship [2016.0123], the Swedish Foundation's Starting Grant (Ragnar Söderberg Foundation), the Swedish Research Council [VR 2016-01842], Karolinska Institutet (SciLifeLab Fellowship, SFO, KID and KI funds) and a Joint China-Sweden mobility Grant (STINT, CH2018-7750). Yin laboratory is supported by 2020 LiaoNing Province Key Research Project (1580441949000), Ganzhou COVID-19 Emergency Research Project, Key Special Project of "Technology Boosts Economy 2020" of Ministry of Science and Technology (SQ2020YFF0411358) for COVID-19 related work.

\section{Competing interests}

VP was a shareholder at Colorna AB. X. Yin is the co-founder of Biotech \& Biomedicine Science (Shenyang) Co. Ltd. S. Ye and X. Liu are employees of Biotech \& Biomedicine Science (Shenyang) Co. Ltd. All other authors declare no potential conflict of interest..

\section{Additional information}

Supplementary Information The online version contains supplementary material available at https://doi. org/10.1038/s41598-020-80352-8.

Correspondence and requests for materials should be addressed to V.P.

Reprints and permissions information is available at www.nature.com/reprints.

Publisher's note Springer Nature remains neutral with regard to jurisdictional claims in published maps and institutional affiliations.

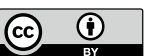

Open Access This article is licensed under a Creative Commons Attribution 4.0 International License, which permits use, sharing, adaptation, distribution and reproduction in any medium or format, as long as you give appropriate credit to the original author(s) and the source, provide a link to the Creative Commons licence, and indicate if changes were made. The images or other third party material in this article are included in the article's Creative Commons licence, unless indicated otherwise in a credit line to the material. If material is not included in the article's Creative Commons licence and your intended use is not permitted by statutory regulation or exceeds the permitted use, you will need to obtain permission directly from the copyright holder. To view a copy of this licence, visit http://creativecommons.org/licenses/by/4.0/.

(c) The Author(s) 2021 